\title{
Comparison of tumour markers in malignant mesothelioma and pulmonary adenocarcinoma
}

\author{
AR GIBBS, R HARACH, JC WAGNER, B JASANI \\ From the Pathology Department, Welsh National School, Cardiff, and the Medical Research Council \\ Pneumoconiosis Unit, Llandough Hospital, Penarth, Glamorgan
}

\begin{abstract}
Immunohistological methods were used to investigate the presence of carcinoembryonic antigen, $\beta_{1}$ pregnancy specific glycoprotein, $\beta$ subunit of human chorionic gonadotrophin, human placental lactogen, calcitonin, and keratin in formalin fixed tissue from 29 malignant mesotheliomas and 27 pulmonary adenocarcinomas. Malignant mesotheliomas were negative for tumour markers except for the $\beta$ subunit of human chorionic gonadotrophin and keratin, one and 13 cases respectively being positive for these. Pulmonary adenocarcinomas, however, were frequently positive for tumour markers - namely, carcinoembryonic antigen (24), $\beta_{1}$ pregnancy specific glycoprotein (23), $\beta$ subunit of human chorionic gonadotrophin (8), human placental lactogen (2), calcitonin (3), and keratin (12). The presence of carcinoembryonic antigen and $\beta_{1}$ pregnancy specific glycoprotein within an intrathoracic tumour is strong evidence against its being of mesothelial origin.
\end{abstract}

The accurate diagnosis of malignant tumours is an essential part of the management of patients, but for some tumours it can still pose many problems. One particularly difficult and important problem is the separation of malignant mesothelioma from pulmonary adenocarcinoma-difficult because these tumours may closely resemble each other in their pattern of spread and their microscopic appearances, ${ }^{12}$ and important not only for clinical management but also for reasons of industrial compensation.

Many attempts have been made to provide an easily used technique which gives a clear separation between the two tumours. Mucin histochemistry has been used, positive staining of tumour cells for hyaluronic acid favours a diagnosis of malignant mesothelioma, whereas the presence of neutral mucin is indicative of adenocarcinoma. ${ }^{34}$ This technique is not, however, widely used, partly because not every adenocarcinoma contains neutral mucin, but also because the preservation of intracellular hyaluronic acid requires special fixative methods. Ultrastructural features can also be used to distinguish these two tumours. ${ }^{5}$ Here too the problem

Address for reprint requests: Dr AR Gibbs, Pathology Department, Welsh National School of Medicine, Cardiff CF4 4XN.

Accepted 3 September 1984 needs to be recognised well in advance, so that the appropriate fixation and embedding techniques can be used.

The introduction of immunocytochemical techniques into routine pathological diagnosis has led to improvement in tumour diagnosis generally; in the differential diagnosis of malignant mesothelioma and adenocarcinoma reports have been conflicting. Antibodies to carcinoembryonic antigen and keratin have been used, and it has been claimed that carcinoembryonic antigen is frequently present in pulmonary adenocarcinoma but almost invariably absent in malignant mesothelioma. ${ }^{6-9}$ Keratin proteins are found by some workers to be frequently present in malignant mesothelioma and absent in pulmonary adenocarcinoma, ${ }^{910}$ while others have found positive keratin staining in pulmonary adenocarcinoma also. ${ }^{1112}$

In a previous study of lung tumours using immunocytochemical techniques we studied various antigens and found that immunoreactive carcinoembryonic antigen was present in $71 \%, \beta_{1}$ pregnancy specific glycoprotein $\left(\mathrm{SP}_{1}\right)$ in $68 \%, \beta$ subunit of human chorionic gonadotrophin ( $\beta \mathrm{HCG})$ in $36 \%$, human placental lactogen in $20 \%$, and calcitonin in $14 \% .{ }^{13}$ Because of the conflicting results of other studies in the separation of pulmonary adenocarcinoma and mesothelioma, we have applied these five antibodies together with a keratin 
antibody to a new series of 56 pulmonary tumours to define which investigations are likely to be of value in this important differential diagnosis.

\section{Methods}

Sections ( $5 \mu \mathrm{m}$ thick) were taken from formalin fixed, conventionally processed paraffin embedded material. Twenty nine malignant mesotheliomas and 27 pulmonary adenocarcinomas were studied. The malignant mesotheliomas were all from necropsy cases with typical macroscopic and microscopic appearances and without any other probable primary source; they comprised 12 purely epithelial tumours, 14 mixed epithelial and sarcomatous tumours, and three purely sarcomatous tumours. Histochemical staining for neutral mucin (periodicacid-Schiff diastase technique ${ }^{14}$ ) was negative for each malignant mesothelioma. The pulmonary adenocarcinomas were from both surgical (13) and necropsy (14) cases: six were well differentiated, three moderately differentiated, and 11 poorly differentiated; three were solid tumours with mucin content, one was a bronchioloalveolar carcinoma, and three were adenosquamous carcinomas.

The six immunohistological markers were localised by a modification of the DPN-hapten staining techniques, ${ }^{15} 16$ the following dilutions and incubation times being used: (1) carcinoembryonic antigen (Dakopatts), 1:2000 for 15 hours; (2) $\beta_{1}$ pregnancy specific glycoprotein (Dakopatts), $1: 1600$ for 15 hours; (3) keratin (Miles-Yeda), 1:200 for 15 hours; (4) $\beta$ subunit of human chorionic gonadotrophin (Dakopatts), 1:400 for 15 hours; (5) human placental lactogen (Dakopatts), 1:800 for 15 hours; (6) calcitonin 1:200 for 15 hours.

Prior trypsinisation ${ }^{17}$ was carried out for one hour for calcitonin and four hours for $\beta_{1}$ pregnancy specific glycoprotein, the $\beta$ subunit of human chorionic gonadotrophin, and human placental lactogen. Trypsinisation was not performed for carcinoembryonic antigen or keratin. Appropriate positive and negative controls were included with each batch of staining. The dilutions and incubation times were selected as those which gave consistently good staining with a clear background in an appropriate positive control tissue. These were slightly modified from those used in our previous study for consistency and greater reliability. ${ }^{13}$

The degree of staining for the appropriate antigen was graded for each tumour as follows: 0 -negative; + (weak)-less than $5 \%$ of the tumour cells intensely or moderately stained; ++ (moderate)$5-33 \%$ of the tumour cells intensely or moderately stained; +++ (strong)-more than $33 \%$ of the tumour cells intensely or moderately stained.

\section{Results}

The results of staining for each tumour marker are shown in the table. Carcinoembryonic antigen was present in 24 out of 27 adenocarcinomas tested but absent in all the malignant mesotheliomas. The tumour product of diaminobenzidine was located predominantly along the luminal surfaces of the tumour cells but it was also seen to a lesser extent in both diffuse and granular patterns within the cytoplasm of the tumour cells, particularly in the less well differentiated adenocarcinomas, but this was not statistically significant. Two of the three adenosquamous carcinomas showed moderate staining for carcinoembryonic antigen; there was staining of both the glandular and the squamous elements, the latter being mainly located along cell membranes and within epithelial pearls.

Staining for $\beta_{1}$ pregnancy specific glycoprotein was positive in 23 of 27 adenocarcinomas but negative in all malignant mesotheliomas tested. There

Results of immunostaining for the six tumour markers

\begin{tabular}{|c|c|c|c|c|c|c|}
\hline \multirow{2}{*}{$\begin{array}{l}\text { Tumour } \\
\text { marker }\end{array}$} & \multirow{2}{*}{$\begin{array}{l}\text { Histological } \\
\text { type of tumour }\end{array}$} & \multicolumn{4}{|c|}{ Degree of positivity* } & \multirow[t]{2}{*}{ Total } \\
\hline & & 0 & + & ++ & +++ & \\
\hline \multirow[t]{2}{*}{ Carcinoembryonic antigen } & Adenocarcinoma & 3 & 8 & 5 & 11 & 27 \\
\hline & Mesothelioma & 29 & 0 & 0 & 0 & 29 \\
\hline$\beta_{1}$ pregnancy specific glycoprotein & Adenocarcinoma & 4 & 10 & 7 & 6 & 27 \\
\hline \multirow{4}{*}{$\begin{array}{l}\text { The beta subunit of human chorionic } \\
\text { gonadotrophin } \\
\text { Human placental lactogen }\end{array}$} & $\begin{array}{l}\text { Mesothelioma } \\
\text { Adenocarcinoma }\end{array}$ & $\begin{array}{l}29 \\
17\end{array}$ & $\begin{array}{l}0 \\
8\end{array}$ & $\begin{array}{l}0 \\
0\end{array}$ & $\begin{array}{l}\mathbf{0} \\
0\end{array}$ & $\begin{array}{l}29 \\
25\end{array}$ \\
\hline & Mesothelioma & 26 & 1 & 0 & 0 & 27 \\
\hline & Adenocarcinoma & 23 & 2 & 0 & 0 & 25 \\
\hline & Mesothelioma & 27 & $\mathbf{0}$ & 0 & $\mathbf{0}$ & 27 \\
\hline Calcitonin & Adenocarcinoma & 22 & 3 & 0 & 0 & 25 \\
\hline \multirow{2}{*}{ Keratin } & $\begin{array}{l}\text { Mesothelioma } \\
\text { Adenocarcinoma }\end{array}$ & $\begin{array}{l}27 \\
15\end{array}$ & $\begin{array}{l}0 \\
7\end{array}$ & $\begin{array}{l}\mathbf{0} \\
\mathbf{3}\end{array}$ & $\begin{array}{l}\mathbf{0} \\
\mathbf{2}\end{array}$ & $\begin{array}{l}27 \\
27\end{array}$ \\
\hline & Mesothelioma & 16 & 8 & 2 & 3 & 29 \\
\hline
\end{tabular}

${ }^{*} 0-$ negative; $+\ldots<5 \%$ of tumour cells intensely or moderately stained; $++-5-33 \%$ of cells intensely or moderately stained; $+++\rightarrow 33 \%$ of cells intensely or moderately stained. 
was no correlation between the histological differentiation of the adenocarcinomas and the degree of positivity. The pattern of staining was similar to that of carcinoembryonic antigen, being strongest along the luminal border of the cells but also present within the cytoplasm in diffuse and granular patterns. Two of the three adenosquamous carcinomas showed weak staining in both the glandular and the squamous components. The latter showed staining of the cell borders and epithelial pearls.

It is noteworthy that staining for either carcinoembryonic antigen or $\beta_{1}$ pregnancy specific glycoprotein or both was positive in all adenocarcinomas but was negative for both in all malignant mesotheliomas. Weak positive staining for the $\beta$ subunit of human chorionic gonadotrophin was observed in eight of 25 adenocarcinomas and one of 27 malignant mesotheliomas. The staining was predominantly intracytoplasmic in diffuse or granular patterns.

Weak positive diffuse intracytoplasmic staining for human placental lactogen was seen in two of 25 adenocarcinomas, while it was negative in all 27 malignant mesothelioma.

Staining for calcitonin was weakly positive in three of 25 adenocarcinomas; the three positives comprised a solid carcinoma with mucin content, a poorly differentiated acinar adenocarcinoma, and a moderately differentiated papillary adenocarcinoma. It was negative in all 27 malignant mesotheliomas.

Positive staining for keratin was observed in 12 of 27 adenocarcinomas and in 13 of 29 malignant mesotheliomas, but there was no direct correlation with differentiation. In the adenocarcinomas staining was seen predominantly at the periphery of the cells. The malignant mesotheliomas showed mainly diffuse intracytoplasmic staining, present in both epithelial and sarcomatous areas but more prevalent in the former.

There appeared to be no significant differences in the staining of any one marker between necropsy and surgical tissues.

\section{Discussion}

Our study clearly shows that the use of immunolocalisation procedures for certain antigens is a valuable adjunct in the differential diagnosis of malignant mesothelioma from pulmonary adenocarcinoma. The most useful in this respect appear to be carcinoembryonic antigen and $\beta_{1}$ pregnancy specific glycoprotein.

Our findings of carcinoembryonic antigen in $89 \%$ of adenocarcinomas and in none of the malignant mesotheliomas accords with those of other investi- gators. Wang $e^{t} a^{6}$ found carcinoembryonic antigen positivity in all 12 pulmonary adenocarcinomas tested but in none of nine malignant mesotheliomas. Whitaker $e^{t} a^{7}$ found positivity in 22 of 26 pulmonary adenocarcinomas and in none of 43 malignant mesotheliomas. Corson and Pinkus, ${ }^{8}$ however, found weak staining in eight and moderate staining in one of 20 malignant mesotheliomas, whereas they found that all adenocarcinomas exhibited strong or moderate staining. Pascal et al $^{18}$ found positivity in 16 of 22 adenocarcinomas. We conclude that a positive immunoreaction for carcinoembryonic antigen is strong evidence against a mesothelial origin for a primary intrathoracic tumour.

The so called trophoblast specific pregnancy proteins $-\beta_{1}$ pregnancy specific glycoprotein, the beta subunit of human chorionic gonadotrophin and human placental lactogen, normally synthesised by the human placenta - have now been demonstrated by immunolocalisation procedures in tissue sections of certain non-trophoblastic as well as trophoblastic tumours. ${ }^{131920} \mathrm{~A}$ few studies have reported raised serum concentrations of various placental proteins in a small proportion of patients with lung carcinomas. ${ }^{21}{ }^{23}$ Wilson et al ${ }^{24}$ investigated the beta subunit of human chorionic gonadotrophin in tissue sections from 61 lung tumours and found positive staining in $84 \%$. In a previous study using immunolocalisation procedures we showed $\beta_{1}$ pregnancy specific glycoprotein in $68 \%$, the beta subunit of human chorionic gonadotrophin in $36 \%$, and placental lactogen in $20 \% .^{13}$ The present study of a separate group of tumours shows that pulmonary adenocarcinomas are frequently positive for $\beta_{1}$ pregnancy specific glycoprotein (85\%), less frequently for the beta subunit of human chorionic gonadotrophin (32\%), and positive for placental lactogen in only $12 \%$. There was no direct relationship between the presence of one placental protein and the presence of another. With the exception of a case of that showed weak staining for the beta subunit of human chorionic gonadotrophin, malignant mesotheliomas appeared uniformly negative for each of the placental proteins studied. Immunohistological investigation of placental proteins in malignant mesotheliomas has not previously been reported and our results show that the presence of a positive immunoreaction of $\beta_{1}$ pregnancy specific glycoprotein is again strong evidence against a mesothelial origin for a primary intrathoracic tumour.

Raised levels of calcitonin have been described in various lung tumours ${ }^{25-26}$ but few immunohistological studies have reported. Calcitonin positive cells have been described in some bronchial car- 
cinoids, small cell carcinomas, and adenocarcinomas of lung. ${ }^{132-28}$ The present study showed weak positivity in $12 \%$ of pulmonary adenocarcinomas. All malignant mesotheliomas were negative.

Previous results for keratin staining in adenocarcinomas have been conflicting. Corson and Pinkus ${ }^{8}$ stated that staining for keratin was weak or negative in $18 / 20$ pulmonary adenocarcinomas and positive in all 20 malignant mesotheliomas studied. Other workers, however, have reported keratin positivity in appreciable proportion of adenocarcinomas. ${ }^{122930}$ Our results show keratin positivity as frequently in adenocarcinomas $(12 / 27)$ as in malignant mesotheliomas (13/29). The frequency of keratin positivity in our series of malignant mesotheliomas is less than in most other series. ${ }^{-}$This could be accounted for in several ways. It is possible that tumour keratin antigenicity might be lost after death so that our postmortem series might be expected to show a lower incidence of positivity than the reported series using surgical pathological specimens. We found no difference, however, in the incidence of keratin positivity between postmortem and surgical cases of adenocarcinoma. Secondly, some workers have reported better results for keratin staining after fixation in ethanol, methanol, or Carnoy's solution. ${ }^{12}$ Thirdly, results for keratin positivity could vary because cytokeratins are heterogeneous and different antibodies have different specificities against different cytokeratin polypeptides. ${ }^{31}$ More work is needed with monoclonal antibodies to determine whether some are more useful than others in differentiating malignant mesothelioma from pulmonary adenocarcinoma.

The alcian blue staining technique with and without hyaluronidase was not used with these tumours because we, like others, ${ }^{32}$ have found that after formalin fixation hyaluronic acid can be demonstrated in less than half of malignant mesotheliomas. The malignant mesotheliomas chosen for this series were typical in gross and light microscopical appearance with little doubt as the the diagnosis.

Our study indicates that of the six tumour markers investigated carcinoembryonic antigen and $\beta_{1}$ pregnancy specific glycoprotein are the most helpful in the distinction of malignant mesothelioma from pulmonary adenocarcinoma. Carcinoembryonic antigen and the $\beta_{1}$ pregnancy specific glycoprotein were negative in all the malignant mesotheliomas tested whereas one or both were present in all the adenocarcinomas. Thus a positive result for either carcinoembryonic antigen or $\beta_{1}$ pregnancy specific glycoprotein in a tumour is strong evidence against a diagnosis of malignant mesothelioma. A negative result for both carcinoembryonic antigen and $\beta_{1}$ pregnancy specific glycoprotein is highly suggestive of malignant mesothelioma if the histological appearances are compatible. Since antibodies to both carcinoembryonic antigen and $\beta_{1}$ pregnancy specific glycoprotein are readily available as standardised commercial products, the combined immunolocalisation of carcinoembryonic antigen and the $\beta_{1}$ pregnancy specific glycoprotein should prove an efficient routine means of differentiating lung adenocarcinoma from difficult cases of pleural mesothelioma in both necropsy and surgical cases.

We gratefully acknowledge the assistance of Professor ED Williams and Mr ND Thomas, and of Miss J Stitfall for typing the manuscript.

\section{References}

1 Harwood TR, Gracey DR, Yakoo H. Pseudomesotheliomatous carcinoma of the lung. A variant of peripheral lung cancer. Am J Clin Pathol 1976; 65:159-67.

2 McCaughey WTE, Oldham PD. Diffuse mesotheliomas: observer variation in histological diagnosis. In: Bogovski P, Gilson JC, Timbrell V, Wagner JC, eds. Biological effects of asbestos. Lyons: International Agency for Research on Cancer, 1973:58-61.

3 Wagner JC, Munday DE, Harrington JS. Histochemical demonstration of hyaluronic acid in pleural mesotheliomas. J Pathol Bacteriol 1962;84:73-8.

4 Kannerstein M, Churg J, Magner D. Histochemical studies in the diagnosis of mesothelioma. In: Bogovski P, Gilson JC, Timbrell V, Wagner JC, eds. Biological effects of asbestos. Lyons: International Agency for Research on Cancer, 1973:62-4.

5 Wang NS. Electron microscopy in the diagnosis of pleural mesotheliomas. Cancer 1973;31:1046-54.

6 Wang N, Huang S, Gold P. Absence of carcinoembryonic antigen-like material in mesothelioma. An immunohistochemical differentiation from other lung cancers. Cancer 1979;44:937-43.

7 Whitaker D, Sterrett GP, Shilkin KB. Detection of tissue CEA-like substance as an aid in the differential diagnosis of malignant mesothelioma. Pathology 1982;14:255-8.

8 Corson JM, Pinkus GA. Mesothelioma: Profile of keratin proteins and carcinoembryonic antigen. An immunoperoxidase study of 20 cases and comparison with pulmonary adenocarcinoma. Am J Pathol 1982;108:80-7.

9 Said JW, Nash G, Tepper G, Banks-Schlegel S. Keratin proteins and carcinoembryonic antigen in lung carcinoma. Hum Pathol 1983;14:70-6.

10 Schlegel R, Banks-Schlegel S, McLeod JA, Pinkus GS. Immunoperoxidase localisation of keratin in human neoplasms. Am J Pathol 1980;101:41-50.

11 Espinoza CG, Azar HA. Immunohistochemical localisation of keratin-type proteins in epithelial neoplasms. Am J Clin Pathol 1982;78:500-7.

12 Ramaekers F, Puts J, Maester O, Kant A, Jap P, Vooijs P. Demonstration of keratin in human adenocarcinoma. Am J Pathol 1983;111:213-23.

13 Harach HR, Skinner M, Gibbs AR. Biological markers in human lung carcinoma: an immunopathological study of six antigens. Thorax 1983;38:937-41. 
14 Pearse AGE. Histochemistry, theoretic and applied. London: Churchill Livingstone, 1971:660.

15 Jasani B, Wynford-Thomas D, Williams ED. Use of monoclonal antihapten antibodies for immunolocalisation of tissue antigens. J Clin Pathol 1981;34:1000-2.

16 Jasani B, Thomas ND, Newman GR, Williams ED. DNP-hapten sandwich staining (DHSS) procedure: Design, Sensitivity, Versatility and Applications. Immunol Commun 1983;12:51.

17 Gibbs AR, Johnston NF, Giddings JC, Jasani B. Primary angiosarcoma of the mediastinum. A light and electron microscopical study with demonstration of Factor VIII related antigen in neoplastic cells. Hum Pathol 1984; 15:687-91.

18 Pascal RR, Mesa-Tejada TR, Bennet SJ, Garces A, Fenoglio CM. Carcinoembryonic antigenimmunohistologic identification in invasive and intraepithelial carcinoma of the lung. Arch Pathol Lab Med 1977;101:568-71.

19 Walker RA. Biological markers in human breast carcinoma. J Pathol 1982;137:109-17.

20 Horne $\mathrm{CHW}$, Nisbet $\mathrm{AD}$. Pregnancy proteins: a review. Invest Pathol 1979;2:217-31.

21 Gropp C, Haremann K, Scheuer A. Ectopic hormones in lung cancer patients at diagnosis and during therapy. Cancer 1980;46:347-54.

22 Rosen SW, Weintraub BD, Vaitukatis JL, Sussmann HH, Hershman JM, Muggier FM. Placental proteins and their subunit as tumour markers. Ann Intern Med 1975;82:71-83.

23 Grudzinskas JS, Coombes RC, Ratcliffe JG, Gordon YB, Powles TJ, Munro Neville A, Chard T. Circulating levels of pregnancy specific $\beta_{1}$ glycoprotein in patients with testicular, bronchogenic and breast carcinoma. Cancer 1980;45:102-3.

24 Wilson TS, McDowell EM, McIntire KR, Trump BF. Elaboration of human chorionic gonadotrophin by lung tumours. Arch Pathol Lab Med 1981;105:169-73.

25 Silva OL, Broder LE, Doppman JL, Snider RH, Moore CF, Cohen MH, Becker KL. Calcitonin as a marker for bronchogenic cancer. Cancer 1974;44:680-4.

26 Milhaud G, Calmette C, Taboulet J, Julienne A, Markhtar MS. Hypersecretion of calcitonin in neoplastic conditions. Lancet 1974; i:462-3.

27 Cooney T, Sweeney EC, Luke D. Pulmonary carcinoid tumours: a comparative regional study. J Clin Pathol 1979;32:1100-9.

28 Dayal Y, O' Brien DS, Wolfe HJ, Reichlin S. Carcinoid tumours: a comparison of their immunocytochemical hormonal profile with morphologic and histochemical characterisation. Lab Invest 1980;42:111 (abstract).

29 Walts AE, Said JW, Banks-Schlegel S. Keratin and carcinoembryonic antigen in exfoliated mesothelial and malignant cells. An immunoperoxidase study. Am $J$ Clin Pathol 1983;80:671-6.

30 Nagle RB, McDaniel KM, Clark VA, Payne CM. The use of antikeratin antibodies in the diagnosis of human neoplasms. Am J Clin Pathol 1983;79:458-66.

31 Muijen GNP, Ruiter DJ, Ponec M, Huiskens-Van May C, Warnaar SO. Monoclonal antibodies with different specificities against cytokeratins. Am J Clin Pathol 1984;114:9-17.

32 Whitaker D, Shilkin KB. Diagnosis of pleural malignant mesothelioma in life-a practical approach. $J$ Pathol 1984;143:147-75. 